\title{
Medical Informatics in Morocco
}

\author{
0. Bouhaddou', M. Bennani Othmani', S. Diouny ${ }^{3}$ \\ ${ }^{1}$ Hewlett Packard (HP), San Diego, California, USA \\ ${ }^{2}$ Casablanca Medical Informatics Laboratories, School of Medicine, Casablanca, Morocco \\ ${ }^{3}$ Chouaib Doukkali University, Faculty of Letters \& Human Sciences, El Jadida, Morocco
}

\begin{abstract}
Summary
Objectives: Informatics is an essential tool for helping to transform healthcare from a paper-based to a digital sector. This article explores the state-of-the-art of health informatics in Morocco. Specifically, it aims to give a general overview of the Moroccan healthcare system, the challenges it is facing, and the efforts undertaken by the informatics community and Moroccan government in terms of education, research and practice to reform the country's health sector.

Methods: Through the experience of establishing Medical Informatics as a medical specialty in 2008, creating a Moroccan Medical Informatics Association in 2010 and holding a first national congress took place in April 2012, the authors present their assessment of some important priorities for health informatics in Morocco.

Results: These Moroccan initiatives are facilitating collaboration in education, research, and implementation of clinical information systems. In particular, the stakeholders have recognized the need for a national coordinator office and the development of a national framework for standards and interoperability.

Conclusion: For developing countries like Morocco, new health IT approaches like mobile health and trans-media health advertising could help optimize scarce resources, improve access to rural areas and focus on the most prevalent health problems, optimizing health care access, quality, and cost for Morocco population.
\end{abstract}

\section{Keywords}

Medical informatics, Morocco, mobile health, developing countries

Yearb Med Inform 2013:190-6

\section{Introduction}

The technologies collectively known as health information technology (health IT) share a common attribute: they enable the secure collection and exchange of vast amounts of health data about individuals. The collection and movement of these data will power the health care of the future. It also has the potential to empower individuals and increase transparency; enhance the ability to study care delivery and payment systems; and ultimately achieve improvements in care, efficiency, and population health [1].

North American and Europe have seen real advances in the understanding of different types of medical informatics applications. The growth of health IT and medical informatics as a discipline are largely due to advances in information technology in general, and to the fact that biomedical records data are now essentially unmanageable by traditional paper-based methods. In addition, health IT has been recognized as an essential tool that can help address the major health challenges most countries face related to inefficient access, quality, and cost. Governments everywhere realize the efficiencies that can be gained and have been making significant investments in health IT. Health care reforms are driving the evolution of the healthcare system toward a digital, data-driven, patient-centered, community-linked, new model of payment-facilitated healthcare system. For instance, legislation in the U.S. has established the Health Insurance Portability and Accountability Act (HIPAA), the Office of the National Coordinator for health IT (ONC), the Health Information Technology for Economic and Clinical Health Act (HITECH) Act, and Meaningful Use [1]. The recommendations from ONC and a recent Institute of Medicine (IOM) report recommend increased adoption and meaningful use, improve individual and population health, inspire trust in health IT, empower individuals to use health IT to improve their health, and improve the health care system - a rapid learning and advancing system [2].

Medical informatics is concerned with the use of information in health care by clinicians. It is at the core, aligning IT with business and clinical objectives. Physicians recognize that. As a result, medical informatics is becoming a medical specialty providing clinicians with career opportunities to teach, do research, and implement IT solutions that are used in a meaningful way to address today's healthcare challenges.

However, if there are advances in specific communities, they have not yet made a general impact. Indeed, these technologies are still under-utilized even in the developing countries. For instance, in 2009 in the US, only $25 \%$ of physicians have adopted Electronic Health Records (EHRs), only $15 \%$ of hospitals have implemented a hospital information system, and only $7 \%$ of patients have accessed their records online $[3,4]$. However, these numbers are drastically increasing as a result of the significant health IT stimulus made as part of healthcare reform ('obamacare'.)

Health IT is beginning to make an impact in developing countries also. In Morocco, progress has been registered in the field of medical informatics in the areas of academic studies, research, and practice over the last several years $[5,6]$. For example, a formal medical informatics laboratory was created in 1997 at the School of Medicine of Casablanca-Morocco, stimulated by several health IT projects initiated in the 1980s in 
Rabat School of Medicine. In the laboratory in Casablanca, a first position for a clinician resident in medical informatics was created. In 2001, the first Assistant Professor position in medical informatics was created at the same school. In 2008, medical informatics was recognized as a medical specialty. There are now several medical doctors doing their residency in medical informatics. In 2009, the Moroccan Medical informatics Association (SMIMS) was created. In April 2012, the first national conference in medical informatics (CNIM12) was organized.

Developing countries can learn from the advances made in medical informatics around the world and adapt to their local situations or leapfrog them. We hope this paper will help promote medical informatics as a new field of interest in Morocco. In a first section, our article gives an overview of the Moroccan health care system with a summary of the challenges it faces and the current informatics efforts in education, research, and practice. Section two introduces the SMIMS and the CNIM12. In section three, we discuss challenges and opportunities for medical informatics in Morocco. In particular, how can Morocco leverage stronger national and international collaborations and translate them into effective education, research, and applications objectives of health IT that benefit Moroccan citizens and the population as a whole.

\section{Moroccan Health Care System}

The Moroccan health system includes a public and private sector, and is currently undergoing a number of reforms, including financial, hospital and institutional reforms.

The public health sector provides $85 \%$ of the country's hospital beds and represents the main employer of health professionals in Morocco. This sector deals particularly with the needs of poor and rural populations who are unable to afford the service offered by the private sector. It is run mainly by government-funded (i.e., social security (called CNSS)) health centers or clinics that are located in every district, and services rendered are free of charge.

The private health sector is a profit-making sector that is principally attended by people with sufficient income or those who have health insurance. It caters for the needs of $16 \%$ of the population covered by health insurance [7].

For the most part, clinicians learn and practice in French in Morocco.

\subsection{Quality and Access to Healthcare Services}

The Moroccan healthcare system has been instrumental in the improvement of the health standards in independent Morocco. For example, the infant mortality rate fell considerably between 1962 and 2004, from 118 to 40 deaths per 1000 live births, while from 1972 to 2004, the maternal mortality rate fell from 631 to 227 deaths per 100000 births. However, this apparent improvement is tempered by significant urban-rural disparities. For example, life expectancy at birth is higher in urban than in rural areas. The infant mortality rate recorded in rural areas is twice that of urban areas and the maternal mortality rate is $30 \%$ higher in rural areas, both infant mortality and maternal mortality constitute a major challenge for the Moroccan health care system. By 1999 , over $93 \%$ of the children up to one year of age were vaccinated against tuberculosis, $87 \%$ were protected against Diphtheria, Pertussis, Tetanus, and Polio. Infant mortality rates have decreased to 36 deaths out of 1000 live births. Life expectancy has increased from 48 years in 1967 to 71.8 years in 2008 . Close to $82 \%$ of the population has access to safe drinking water and $75 \%$ has adequate sanitation. According to World Bank 2004 criteria, Morocco is a lower middle income country.

Despite the Moroccan government efforts to reform the country's health sector, the Moroccan healthcare system suffers from many inadequacies: insufficient human and material means, uneven geographic distribution of health cover and a very limited health insurance. People living in poverty sometimes do not receive the care that adequately responds to their needs.
There are also health care facilities that are not equipped or only partially functional, and there is an under-use of almost the entire health care system (average occupation rate is $56 \%$ ). More than seven in ten Moroccans $(71 \%)$ do not have access to a formal health care provider. The average number of medical consultations per year is 0.6 contacts per person in urban areas and 0.4 contacts in rural areas. Recourse to health care is strongly linked to the distance that must be covered to reach the health care center with the closer the center, the greater the average number of consultations per inhabitant.

Morocco has a high prevalence of specific health problems, in particular in gastro-intestinal diseases, respiratory diseases, hematology disorders. Certain infectious and parasitic diseases are still prevalent like tuberculosis, certain perinatal conditions, and HIV and other sexually transmitted diseases. The Moroccan health care system is addressing these challenges and improvements are noticeable, especially over the last decade.

\subsection{Health Care Cost}

Despite the fact that the financing of the health care system in Morocco increased to a global expenditure of 4.3 billion US Dollars at the end of 2008, namely $5.6 \%$ of the Gross Domestic Product (GDP), it continues to lag behind most of the countries in the region. In 2001, Lebanon allocated the equivalent of $12.2 \%$ of its GDP to fund its health system, $9.5 \%$ in the case of Jordan and $6.4 \%$ for that of Tunisia. Morocco's national healthcare system spends more than $33.6 \%$ of its monetary resources on buying medicine and medical goods. Health promotion check-ups and external consultations total $35.2 \%$ of spending. This spending is exacerbated by the weakness of funds allocated to collective health prevention (drinking water testing, information, education and communication) [7].

Collective financing of health expenses is optional in Morocco and very limited. It mainly covers civil servants, and urban formal sector workers. Its coverage barely exceeds $16 \%$. Direct payments by insurance companies represent roughly $54 \%$ of total health expenditure [7]. 
Morocco allocates fewer funds to its healthcare; its healthcare system is somewhat poorly maintained and lacks the adequate capacity to meet the demands for necessary medical treatments. Also, there is a disparity between urban and rural locations, and between regions: $78 \%$ of the healthcare budget goes towards urban hospitals and clinics, which has left rural medical services difficult to access and in many cases lacking basic medical equipment and medicine. For example, only two regions have two midwives per rural birth centre. In other words, rural and distant areas of Morocco have by and large remained behind the dynamism of the larger cities in terms of economic and human development [8].

\subsection{Moroccan Health System Challenges}

Morocco faces serious health care challeng-

es. These can be identified as follows:

- The first and most important challenge is the low level of the government budget allocated to public health in Morocco. Despite the efforts undertaken by the Moroccan government over the last five years, public hospitals, representing more than $80 \%$ of national bed capacity, only receive $9.8 \%$ of insurance spending in the context of direct payments and 6.6\% of the total health insurance spending [7].

- The second challenge is inequity of public spending. Rural and poor households benefit less from public spending on health compared to urban and better-off segments of the population; hence, the difficulty in accessing health care for the poorest and for rural populations. The level of use of health care services is, in half the cases, linked to financial support (Moroccan Ministry of Health report 2007).

- High cost of private medical care and low coverage of health insurance.

- Lack of regulation in healthcare. Consumers not sufficiently protected against potential abuse.

- Poor management of public hospitals, which suffer from a range of inefficiencies, making them unable to compete with private hospitals. These inefficiencies include:
- Centralized management and lack of autonomy. Lack of transparency

- Lack of a policy to manage and develop human resources, resulting in deficiency in managerial competence, technical resources to operate technical facilities, and problems of absenteeism and corruption

- Poor care quality and patient satisfaction, linked to lack of coordination with primary care provider

- Absence of drug policies leading to insufficient use of generic medicines; and no benchmark for care therefore no clarity in the policy for fixing prices, charging for surgery

- Deficiencies in specialized structures for geriatrics and absence of retirement homes.

\section{Health Informatics in Morocco}

There is a growing interest and activity level in health informatics within university hospitals and medical schools. However, to this date, there is one formal department of medical informatics within a medical school, the one in Casablanca, called Casablanca Medical Informatics Laboratory (CMIL). One author is from this laboratory.

The discipline of medical informatics within the university is defined by an article from the Moroccan Department of Education, Professional Training, and Research (article 1439-99, BO 4736). This article specifies health informatics as a university discipline. As to medical informatics, it has been recognized as a medical specialty by Morocco's secretary of health in July 2008 and is regulated by the official act no. BO 5644, 2008.

\subsection{Education}

Typically, medical students, doctors and other clinicians (e.g., nurses, pharmacists), and others (e.g., librarians, engineers, epidemiologists) who have been interested in health informatics had to self-train or enroll in a Masters/PhD program abroad, mostly in France. At the time of writing of this article, only one laboratory in Morocco - CMIL, a department of the Casablanca School of Medicine and Pharmacy - has been offering medical informatics courses as an integral class within the medical school syllabus, during the 5 th year of a 6 -year program. It is offered within a module called community health. In parallel, the CMIL is responsible for a residency program for physicians interested in the medical informatics specialty. The residency program takes 4 years and requires 114 credit hours of education. The 114 credit hours are comprised of 6 modules: biostatistics (19), epidemiology (26), research methodology (6), information technology (17), information management (4), and health informatics (42). At the end of this residency, candidates are board certified in medical informatics. At this point, there is no health informatics training program offered in Morocco for non-clinicians, except for training seminars that are open to all with topics like introduction to health informatics, biostatistics, research and critical review of medical literature, production of multimedia training material, and others.

\subsection{Research}

The main projects that CMIL is involved in are listed below: International NetAdded Project (new technologies to avoid digital division in e-divided areas) whose purpose is to create research centers to disseminate research results for the implementation of the European Community sixth framework program. International EMISPHER Project (Euro-Mediterranean Internet-Satellite Platform for Health, Education and Research) co-funded by the European Commission in the frame of the program EUMEDIS. It aims at promoting cooperation between Mediterranean countries [6]. Another project involves the setup of a remote clinic that is completely dependent on telemedicine to operate [5].

Several new research projects are being initiated. They investigate innovations that address the problems and challenges raised by the medical community. In particular, 
CMIL is collaborating with several universities including the engineering school of the University of El Jadida, the dental school of Casablanca, and the engineering school of the University of Casablanca. There are also collaboration projects sponsored by the Moroccan Medical Informatics Association (SMIMS), such as the 'e-App' project with an engineering school (Ecole Nationale de l'Industrie Minérale).

\subsection{Practice}

Over the last 4 years, there have been significant investments in clinical information systems made in particular by the 5 university hospitals: University Hospital Hassan II in Fes, University Hospital Mohammed VI in Marrakech, University Hospital Ibn Sina in Rabat, and University Hospital Ibn Rochd in Casablanca. For example, CMIL participated in the elaboration of the health IT Master Plan for Ibn Rochd University Hospital. It provided a strategic vision for all health IT projects. Also, it identified specific requirements and solutions, with an overarching alignment in real-time between the flow of information to support patient care (administrative, medical and nursing) and medical education, with the physical flow of medical materials, medications, blood samples, etc. and activities related to the patients (e.g., prescriptions, exams, medical or surgery acts). The main objectives of these clinical information systems can be grouped in three main categories:

1) Documentation and data recording relative to the various steps of medical care: diagnosis, prescriptions, and results;

2) Information dissemination: health information exchange, e.g., drug prescription must be exchanged with the pharmacy; and

3) Support for administrative and medical decision-making: a dashboard allows analyzing medical activity, costs by patient, stay by patient, and other data analysis metrics.

Also, it is worth noting that there is interest from other stakeholders to be involved in health IT, including pharmaceutical com- panies, service providers, mobile phone operators, university sciences and engineering departments.

\subsection{Moroccan Medical Informatics Association}

The idea of creating a Moroccan association of health informatics was discussed for several years. Typically, these discussions would take place during international meetings where Moroccan nationals and Moroccan experts living abroad would meet. French pioneers, like Professor Marius Fieschi of Marseille University, generously shared their experience and provided excellent recommendations. However, it has been hard to make rapid progress with communication across continents. A tipping point has been when a core team of national experts came together and volunteered their times for the cause. A charter was developed with nomination of the executive office (Professor Bennani being the first president and Dr. Bouhaddou the president honorarium) and the name Moroccan Society of Medical Informatics (SMIMS is the used French acronym). The application for a not-for-profit organization was filed with the Moroccan administration. A web site was created (http:// www.smims.ma) to describe the association goals, inform about upcoming events (e.g., congress in medical informatics), and allow new members to join.

SMIMS offers several education and training certificates, including introduction to medical informatics, fundamentals of biostatistics, aids to literature searches, guides to critical review of scientific evidence, multimedia presentations, and others. The complete list of SMIMS offerings is published on its web site. Also, SMIMS has organized several training workshops for medical residents and physicians of the public health sector.

\subsection{First National Conference in Health Informatics}

The Moroccan Society of Medical Informatics held its first national conference in Casablanca, April 6-7, 2012. The prepara- tion of this first conference was naturally a challenge for the new SMIMS team. However, when essential elements came together, including the support of key leaders at the University of Casablanca and Ministry of Health, the conference site (given the low budget, it could only be a public site free-of-charge), the engagement of key speakers from Morocco, France and the USA, and the financial support of the private sector (pharmaceuticals and health IT vendors). Communication about the conference was mainly through SMIMS web site, personal contact lists, and a letter to administration offices at several medical and engineering schools.

The aim of this-two-day conference was two-fold: First, to discuss current developments of medical informatics in Morocco, and second, to address local issues in health information systems. The Conference brought together industry leaders, decision makers, educators and professors in the fields of healthcare, biomedical sciences, life sciences, pharmaceuticals, informatics and information technology from Morocco, France and the USA. Over 100 professionals attended the conference with a majority of clinicians. Women were well represented. Over the course of two stimulating days, professionals shared research advances, information trends and new enabling technologies that are driving healthcare management, biomedical research, and healthcare delivery to new heights. The National Medical Informatics Conference 2012 featured five in-depth half-day workshops providing tutorials and dynamic discussions with leading experts in healthcare and biomedical informatics.

The main recommendations that emerged from this national conference are as follows:

- Health informatics is becoming increasingly important to effective and efficient health care;

- To better integrate information system, sharing and exchanging information is necessary;

- The use of information system requires the collaboration and involvement of all stakeholders (i.e. University Hospitals, Ministry of Health, decision makers, professionals and the private sector);

- Medical informatics is an emerging 
discipline, with emerging career opportunities. Therefore, more support should be provided to support this emerging discipline; there is a need for interactions among a wide range of people, including, health professionals, IT professionals, educators and administrators, who are, in many instances, playing pivotal roles as information systems are developed, implemented, adopted, and maintained;

- Development of plans for the introduction of medical informatics in the whole health system to cover education, research, and meaningful use in practice;

- Allocation of financial and human resources to support health informatics plans. Promotion of public awareness to sensitize health care professionals and consumers;

- Development of a Moroccan Framework of Reference for standards and interoperability for health IT.

SMIMS stated its commitment to be the home of clinical and non-clinical subject matter experts in the field of medical informatics. One regular activity for the association is to coordinate a bi-annual national congress. The congress will help showcase advances, identify common challenges and spur collaboration.

SMIMS already announced the second national congress in medical informatics in May 2014 in Fes, Morocco. For Moroccan informaticians, it will be an opportunity to meet local and international experts and develop relationships and collaborations. It will also be an opportunity to recognize local pioneers and centers of excellence, and thus, stimulate best practices in the country.

\section{Challenges and Oppor- tunities for Informatics in Morocco}

In this section, the authors reflect back on the informatics challenges and opportunities in Moroc$\mathrm{co}$, on the lessons learned from the first national congress in medical informatics (CNIM12) and present their own, subjective assessment of some important priorities for the field.

\subsection{National Collaborations}

During CNIM12, the majority of the presentations were from the 5 academic health care organizations and they clearly showed the need for collaboration among these organizations. There were instances where two or more university hospitals were developing software requirements for similar products but were not aware of each other efforts. In another example, one University Hospital had already deployed and addressed user adoption challenges whereas another was about to deploy the same product but did not know how to make a good first impression with its users and avoid resistance to change.

Perhaps, the development of a shared website where organizations could post their projects and lessons learned would help. Tools, processes, people skills information categories could be posted to promote collaboration and pull resources together to achieve greater successes. This would not hamper innovation, but rather bring together teams to solve common problems more efficiently and more rapidly.

Further, collaboration would be greatly facilitated if there was an office for a national coordinator for health IT projects, similar to the US (ONC) [1] or French (ASIP) models. This office would help all organizations, public and private, adopt common standards for health IT so that information can move with patients as they move from provider to provider, can be shared between public to private facilities, and can be re-used effectively for population health. Standards are lacking and are needed for many entities including identifiers for patients, providers, and facilities; structured medical terminologies; security, privacy and consent management, and others.

For instance, the availability of a national patient identifier strongly authenticated to each Moroccan citizen would greatly enhance information sharing and security. Many studies have documented this [9]. A Moroccan collaboration needs to put forth the policies, standards, and incentives to drive development and adoption of this critical infrastructure element.

Eventually, working collaboratively with public and private sectors, the office of Morocco national coordinator would define a national health IT agenda and strategic plan. This will set a timeline for the realization of the major initiatives needed by the country. It will also help develop synergetic resources related to this important transformation, including policies, finances, workforce development and change management.

\subsection{International Collaborations}

In our increasingly global and inter-related community, the opportunities for international collaboration abound. Morocco has and will continue to leverage this positive effect of globalization and open access to information.

Health IT collaboration covers education, research and implementation projects. Historically, the main international ties have been with France. Online Master degrees in Health Informatics in France offer stipends to French-speaking applicants who can take the course from their country and only come to France for the final examination. For instance, in 2010, many Moroccan students completed successfully a Master's in medical informatics Online Graduate Program elaborated by the University of Marseille, France, and coordinated by Professor Marius Fieschi. In addition, the Summer School of Corte in Corsica, France has also been offering training opportunities for many Moroccan informatics specialists, including one of the authors.

Since 1988, French speaking countries have been organizing Medical informatics study days in French-speaking countries. The aim of these scientific seminars is to discuss the state of the art in medical informatics. This congress is held every 2 years in a different French-speaking country. The proceedings are published by Springer. This year, the group has joined the International Medical Informatics Association (IMIA) Special Interest Group, and will benefit from IMIA's experience and outreach capabilities.

Joint projects can bring the strength of multi-cultural students/faculty teams to work on Moroccan projects. Attempts to win grant support from USAID, The Millennium Challenge Corporation and others are underway in Morocco. To expand beyond the North-South traditional allianc- 
es, SMIMS Board is trying to engage other organizations in sharing their experience. In particular, a few months back, SMIMS reached out to the American Medical Informatics Association (AMIA) after it created its Global Health Informatics directorate, inquiring about ways AMIA can be involved in Morocco but has not received a reply yet. Also, through membership with IMIA, SMIMS hopes to shorten the path to productive international collaborations by leveraging IMIA's rich network of medical informatics specialists.

\subsection{The Exciting Opportunity of Mobile Health}

It is predicted that by $2013,95 \%$ of the entire world will have access to cell phones. If a small village in Morocco has only 1-2 phones, the technology is still present. The Mobile Health - revolution can have a tremendous impact on developing countries. Mobile Health is a game changer. As mobile telecommunication allowed developing countries to leap developed countries in terms of social communication outreach and networks, it can also help developing countries significantly advance their health IT [10]. It helps them extend their resources. For instance, the ratio of doctors to individuals is very low in Morocco. Mobile Health, with simple text-based messages can help close this health care gap.

In South Africa, HIV awareness text messages produced a four-fold increase in calls to the national AIDS line. Peru has a program where AIDS patients get a text message reminding them to take their medicine. Smart phones are revolutionizing Africa healthcare [12]. Ugandan software developers are designing an app that could radically change antenatal care in remote areas and save hundreds of lives.

In 2011, the U.S. Department of Health and Human Services announced two new mobile phone text messaging smoking cessation programs, QuitNowTXT and SmokeFreeTXT, as part of their Text4Health initiative. Johnson \& Johnson has a program, Text4Baby that provides education to pregnant women and new parents for 20 million users around the world.
Transmedia is a mass communication approach where integral elements of a story get dispersed across multiple delivery channels for the purpose of creating a unified and coordinated experience and impact. Marketeers use this approach in a diverse array of industries, in particular entertainment. A movie will be released with a comic book, animated cartoons, and a video game. The health industry is starting to embrace transmedia storytelling techniques. For example, Walt Disney made a cartoon movie about malaria and mosquitoes. Also in recent years, health advertising has been used with tremendous results to discuss the effect of smoking, alcohol, weight management, STDs, non-saturated fats on health.

The broad availability of mobile devices can help public health programs reach both providers and consumers, and be effective for individuals and population health, for disease management and for prevention and risk reduction campaigns. Furthermore, there are many resources available to guide this effort. For instance, the US National eHealth Collaborative Patient Engagement Framework is a model created to guide healthcare organizations in developing and strengthening their patient engagement strategies through the use of eHealth tools and resources [12]. The five steps or phases to engagement that make up the framework include "inform me," "engage me," "empower me," "partner with me," and "support my e-community." To realize the benefits of this exciting opportunity, a country must develop a central strategy and central team that will develop the network and communication within a framework that will emphasize standards for data acquisition and information communication.

\subsection{Workforce Development}

The transformation of the health sector from a paper to an electronic industry will require a significant increase in skilled labor, knowledgeable about healthcare, information, communication, and technology.

Health IT workforce development is a broad effort targeting candidates with multiple backgrounds (e.g., technical and clinical) and expertise at multiple levels (e.g., certificates to $\mathrm{PhDs}$ ).
Medical informatics is already a medical specialty in morocco. In 2008, legislation was passed to make medical informatics a medical specialty in Morocco. The fist lab providing formal residency for this specialty is in Casablanca School of Medicine, under the supervision of Professor Bennani. There are currently 5 residents in the program, with the first graduate to be delivered by 2014 . Having physician specialists in medical informatics will provide a critical component of this work force. These specialists will participate and often lead the efforts of acquisition or development, implementation, evaluation, and continuous improvement of electronic health records. In particular, they will help analyze and refine clinical processes that yield quality outcomes.

The challenges ahead are twofold: 1) develop career opportunities for the new physician specialists in medical informatics, and 2) develop a formal examination and preparation program for medical informatics specialists. AMIA in the US took the lead in developing core content for this subspecialty, and published an outline in 2008 [13]. This core content includes four major categories: fundamentals, clinical decision making and care process improvement, health information systems, and leadership and management of change. In parallel, education and communication efforts must be made to accelerate the recognition of this specialty throughout the health care sector. Ministry of Health, public and private health care organizations, medical schools, and industry partners should recognize the merit that health IT specialists in general and clinical informaticists in particular can bring to the success of health care IT knowledge and use in their settings.

SMIMS will help promote informatics education and certification, through local efforts and international collaboration.

\section{Conclusions}

Informatics is an essential tool for helping to transform healthcare from a paper-based to a digital sector. Public and private investments in health informatics education and research, as well as in support of practical implemen- 
tation projects are necessary to develop the necessary workforce and infrastructure to address the difficult problems and roll out products that are usable and useful to improve patient outcomes.

The Moroccan healthcare system suffers from insufficient budget, an uneven distribution favoring urban areas, and poor management and workforce skills. Lessons learned from and collaboration with developed countries can help developing countries like Morocco leverage existing advances in health informatics to address these challenges. In the last 2 decades, Morocco has made significant progress that parallel the technology advances in other domains, including electricity, internet, mobile phones, hospitals networks, and others. Medical informatics is now a medical specialty in Morocco and already several physicians are engaged in residencies that will provide them with the in-depth knowledge and experience to help lead efforts in their health care organizations and at the national level. Medical students are taught basic computer skills, critical literature review, and biostatistics. A two year old Moroccan Medical Informatics Association (SMIMS) is promoting health IT education and collaboration among clinical and non-clinical specialist of health IT. SMIMS inaugural national congress on health informatics took place April 2012 and the next one has already been announced for 2014 .

More national coordination and collaboration are needed to realize the promises of a digital healthcare system in Morocco.
The creation of a national office or committee is essential to help with standards, interoperability, and facilitate health IT adoption with providers and consumers. This office will help identify priorities and provide policy and grant support to address national complex problems, like development of a national patient identifier, or a security framework, or a national Mobile Health program. Also, international partners and collaborations should be actively sought and can be found through programs like IMIA and 'informaticists without borders'.

In particular, for developing countries like Morocco, new health IT approaches like mobile health and transmedia health advertizing could help optimize scarce resources, improve access to rural areas and focus on the most prevalent health problems, optimizing health care budget for better access, quality, and cost to all Moroccan citizens.

\section{References}

1. U.S. Office of the National Coordinator for Health IT strategic plan http://www.healthit.gov/ policy-researchers-implementers/health-it-strategic-planning. Last accessed on Nov 25, 2012

2. Institute of Medicine Report: Best Care at Lower Cost: The Path to Continuously Learning Health Care in America. http://iom.edu/Reports/2012/ Best-Care-at-Lower-Cost-The-Path-to-Continuously-Learning-Health-Care-in-America.aspx. Visited on Nov 25, 2012.

3. Murphy J. State of the Association slides AMIA Chicago 2013 keynote presentation .http://www. amia.org/amia2012/updates. Last accessed on Feb 10,2013
4. ONC Data Brief AHA 2011. http://www.healthit. gov/media/pdf/ONC_Data_Brief_AHA_2011.pdf. Last accessed on Feb 10, 2013

5. Bennani Othmani M, Diouny S, Boumalif O. The Application of a Telemedicine Management System: The Case of a Moroccan Hospital Unit. Assistive Technology 2012;24(3):209-13.

6. Bennani Othmani M, Diouny S, Balar K. Medical informatics in Morocco: Casablanca Medical Informatics Laboratory. Yearb Med Inform 2007;138-40.

7. Country health information, World Health Organization (WHO), http://www.who.int/nha/ country/en. Last accessed on Nov 25, 2012

8. World Health Organization (WHO), http://www. who.int/workforcealliance/knowledge/PPE_Morocco_CaseStudy.pdf. Last accessed on Nov 25, 2012

9. Dick RS, Steen EB, Detmer D, editors. Institute Of Medicine. The computer-based patient record. Washington: National Academy Press; 1997.

10. Kahn JG, Yang JS, Kahn JS. 'Mobile' health needs and opportunities in developing countries. Health Aff 2010;29(2):252-25.

11. Simon SK, Seldon HL. Personal health records: mobile biosensors and smartphones for developing countries. Stud Health Technol Inform 2012;182:125-32.

12. US National eHealth Collaborative - Patient Engagement Framework. http://www.nationalehealth. org/patient-engagement-framework. Last accessed on Nov 252012.

13. Gardner RM, Overhage JM, Steen EB, Munger BS, Holmes JH, Williamson JJ, et al. Core content for the sub-specialty of clinical informatics. J Am Med Inform Assoc 2009;16(2):153-7.

Correspondence to:

Omar Bouhaddou

PO Box 3351

Rancho Santa Fe, CA 92067

USA

Tel (cell): + 18017553263

E-mail: omar.bouhaddou@hp.com 